(Aus der Universitäts-Frauenklinik München. Director:

Geheimrath v. Winckel.)

\title{
Ueber die durch intrauterine Gehirnhämorrhagien entstandenen Gehirndefecte und die Encephalitis congenita.
}

\author{
Von \\ Privatdocent Dr. Ludwig Seitz, \\ Oberarzt der Klinik.
}

(Mit 3 Textfiguren.)

Liest man die Litteratur über die durch angeblich congenitale Encephalitis entstandenen Zerstörungen des Gehirns aufmerksam durch, so kann man sich des Eindruckes nicht erwehren, dass hicr Dinge zusammengeworfen sind, die durchaus verschiedenartig sind. Diese Unklarheit erklärt sich zum Theil durch die Seltenheit der Beobachtung, zum Theil daraus, dass die Beobachtungen meist Jahrzehnte lang zurückliegen.

Es erscheint mir daher gerechtfertigt, hier über einen Fall einer weitgehenden angeborenen Zerstörung der Gehirnsubstanz zu berichten, zumal derselbe geeignet erscheint, in die Genese der interessanten Anomalie ein neues Licht zu werfen.

Dienersb., Köchin, 29 J., III p., Aufnahme 1. 12. 1906.

1. Geburt vor 9 Jahren, Kind lebt und ist gesund. Im April 1905 Abortus im 4. Monat, der angeblich nach Ueberheben eintrat.

3. Schwangersehaft: Kurze Zeit, als die sonst regelmässige Periode ausgeblieben war, fiel P. beim Reinigen der Fenster vom 1. Stocke auf den Boden des Hofraumes, zuerst auf die Füsse und sank dann in die Knie. Es ging daraufhin Blut aus den Genitalien ab, doch nicht sehr viel; sonst trug sie keinen Schaden davon; auch dauerte die Schwangerschaft weiter.

Im 4. Monat der Schwangerschaft stürzte P. ein zweites Mal und $z$ war 3 Stufen tief in den Keller hinab. Sie trug eine sehr grosse und schwere Schïssel mit Fleisch und fiel mit dem Leibe direct a uf die Schüssel. Unmittelbar nach dem Falle ging sehr viel BIut 
aus den Genitalien àb und P. hatte wehenartige Schmerzen. Sie

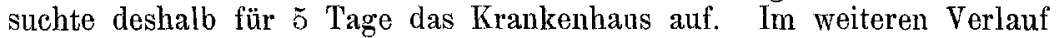
der Schwangerschaft stellten sich wiederholt Blutabgänge aus den Geschlechstheilen ein, so alle 14 Tage bis 3 Wochen; namentlich soll sich nach Heben schwerer Gegenstände Blut gezeigt haben. Die letzte Blutung fand Mitte Oktober, also $1 \frac{1}{2}$ Monate vor der Niederkunft, statt. Kindsbew egungen, die sie vorher gut gefühlt haben will, spürt sie seit einigen Tagen nur sehr wenig mehr. Sie wurde von der Geburt überrascht, da sie die Niederkunft erst Mitte Januar erwartete.

Sonst hat ihr während der Schwangerschaft nie etwas gefehlt, keine Angina u. dergl. Von Lues ist weder anamnestisch noch objectiv etwas nachzuweisen; auch der Kindsvater giebt an, nie Lues gehabt zu haben.

Von Nerven- und Geisteskrankheiten in ihrer Familie weiss sie nur anzugeben, dass 1 oder 2 Kinder der Schwester ihrer Mutter geistig sich schlecht entwickelten; sonst bestanden keine Anomalien.

Part. kommt mit kräftigen Wehen in die Klinik; bevor sie ausgezogen ist, springt die Blase. Die Wehen hatten in geringem Grade bereits 11 Stunden gedauert. Die Herztöne des Kindes sind 120, es wird an ihnen nichts Auffälliges bemerkt. 15 Minuten nach dem Blasensprung erfolgte die spontane Geburt eines im ersten Grade asphyktischen Mädehens, $45 \mathrm{~cm}$ lang, $2800 \mathrm{~g}$ schwer, Kopfumfang suboce. bregm. $27,5 \mathrm{~cm}$, frontooccip. $29,5 \mathrm{~cm}$, grosser querer $8 \mathrm{~cm}$, kleiner querer Durchmesser $6, \tilde{5} \mathrm{~cm}$. Die Nabelschnur war nicht umschlungen, Meconium war unter der Geburt nicht abgegangen, auch bei dem Austritt des Kindes entleerte sich kein Meconium.

Die Wiederbelebung des Kindes dauerte auffällig lange. (Schultze'sche Schwingungen, Trachealaspirationen, thermische Reize.) Erst nach 20 Minuten beginnt das Kind leise zu schreien und flach zu athmen. Es wurde die künstliche Atmung noch weiter fortgesetzt, jedoch trat kein kräftiges Schreien ein. Kind kommt in das v. Winkel'sche Dauerbad.

Die Nachgeburt wurde spontan ausgestossen, wies am Rande einen grossen Infarkt auf und mehrere fibröse Schwarten am Rande und in der Mitte, ihre Grösse war 19:20 cm, $2 \mathrm{~cm}$ dick, das Gewicht $770 \mathrm{~g}$, mit einer Apoplexie.

Die Mutter machte ein fieberfreies Wochenbett durch, der Uterus bildete sich gut zurück, Störungen von Seiten irgend eines Organs liessen sich nicht nachweisen, am 7. Tage verliess sie gesund die Klinik.

Das Kind erholte sich auch im Dauerbad nicht recht, die Athmung blieb oberflächlich; Krämpfe traten keine auf, auch keine 
Zuckungen im Gesicht, auch fiel an der Haltung des Körpers nichts besonderes auf. 5 Stunden nach der Geburt starb es.

\section{Sectionsbericht.}

Starke Cyanose der Haut. Beide Lungen fast völlig atelektatisch. Mittel- und Unterlappen völlig luftleer. Gewebe schneidet sich leberartig.

Oberlappen enthalten etwas Luft.

In den grossen Bronchien sowie in der Trachea kein Schleim und keine anderen fremden Bestandtheile.

Herz: Klappen intakt, Foramen ovale noch offen; sonst kein besonderer Befund.

Leber: Von eigenthümlich schlaffer Consistenz, etwas vergrössert.

Milz: Etwa um die Hälfte der normalen vergrössert. Malpighi'sche Körper treten sehr deutlich hervor. Trabekel gut sichtbar.

Massenhaft freier Ascites, auch imHerzbeutel, sowie in beiden Pleurahöhlen etwas wässerige klare Flüssigkeit.

\section{Beschreibung des Kopfes.}

Der Kopf hat einen frontooccipit. Umfang von $29 \mathrm{~cm}$, suboccipitobregmatical. Umfang von $28 \mathrm{~cm}$. Das Gesicht zeigt regelmässige Entwicklung, ebenso Nase, Mund, Rachen, Augen. Die Schädelknochen sind regelmässig gebaut, gut verknöchert, zeigen nirgends Ossificationsdefecte. Pfeilnaht hat eine Breite von $1 \mathrm{~mm}$. Grosse Fontanelle recht klein. Stirnbeine sind etwas unter dem Scheitelbeine verschoben. Stärker ist die Verschiebung des Hinterhaupts unter die Parietalia. Nach Abziehen der Kopfschwarte zeigt sich stärkere serös-sanguinolente Durchtränkung über dem Hinterhauptbein von der Kopfgeschwulst herrührend. Ueber allen Knochen des ganzen Craniums sind einzelne kleinere Hämorrhagien zerstreut. Die grosse Fontanelle fühlt sich etwas gespannt an.

Nunmehr wird der Schädel so eröffnet, dass in die Pfeilnaht mit der Scheere möglichst lateral eingestochen und das Scheitelbein durch Fortsetzung des Schnittes nach der Kranz- und Lambdanaht sammt der Dura nach unten zurückgeklappt wird. Dabei ergiesst sich aus der Schädelhöhle eine reichliche, etwa $1 / 4$ Liter betragende Flüssigkeitsmenge, die fast klar, etwas zähflüssig ist und eine leicht gelbliche Beschaffenheit zeigt. Blutbeimengung ist 
nicht vorhanden, auch keine Gewebsfetzen finden sich in der Flüssigkeit. Leider wurde versäumt, die Flüssigkeit aufzufangen und sie chemisch-mikroskopisch zu untersuchen. Ebenso wurde keine Cultur angelegt.

In gleicher Weise wurde das andere Scheitelbein eröffnet, so dass in der Mitte nur noch eine $2 \mathrm{~cm}$ breite den Sinus longitud. tragende Membran erhalten war.

Ueber die Ausdehnung der Zerstörung des Grosshirns giebt am besten ein Sagittalschnitt durch den Kopf Aufschluss. Man sieht

Figur 1.

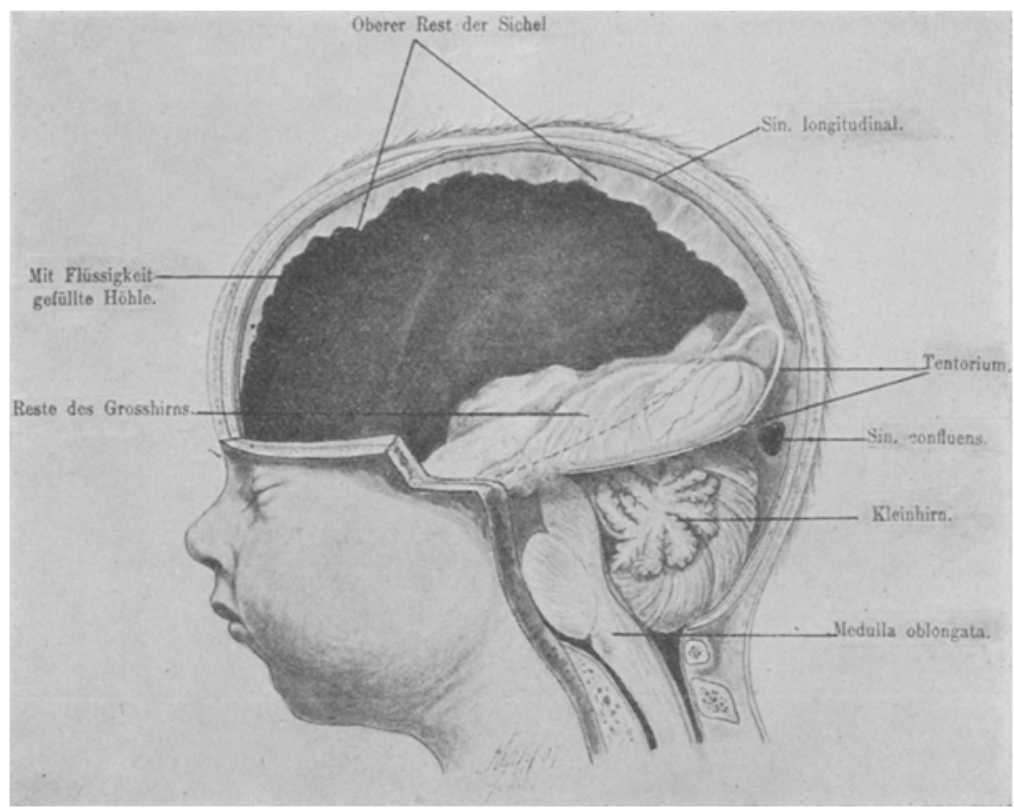

Sagittalschnitt durch den Kopf.

an der Fig. 1, dass vom Grosshirn Stirn- und Scheitellappen vollständig" fehlen und dass vom Occipitallappen nur der hintere Theil und vom Temporallappen ein Stück vorhanden ist. Die Aussenfläche der noch erhaltenen Lappenpartien zeigt eine gute Ausbil. dung der Furchen und Windungen, regelmässige Blutgefässversorgung, gewöhnliche Farbe, normales Verhalten der Meningen. Das Tentorium ist regelmässig ausgebildet, Kleinhirn und Medulla oblongata zeigen normale Verhältnisse. Man sieht auf dem Schnitte 
den Sinus confluens getroffen, der gute Entwicklung und keine Thrombosirung zeigt. Auch der Sinus longitudinalis ist durchgängig, doch ist sein Lumen entschieden etwas kleiner als sonst.

Von grossem Interesse ist das Verhalten der Höhle.

Die Sichel, die unter normalen Verhältnissen mehrere Centimeter tief $z$ wischen die Hemisphären hereinreicht, ist bis auf einen oberen und unteren Rest zerstört: der obere (Fig. 1) zeigt einen unregelmässigen, fetzigen Rand, der Sinus longitudinalis ist erhalten, aber schmäler als sonst, der untere Rest (Fig. 2) zieht als dünner Strang nahe der Schädelbasis von der Crista galli über

Figur 2.

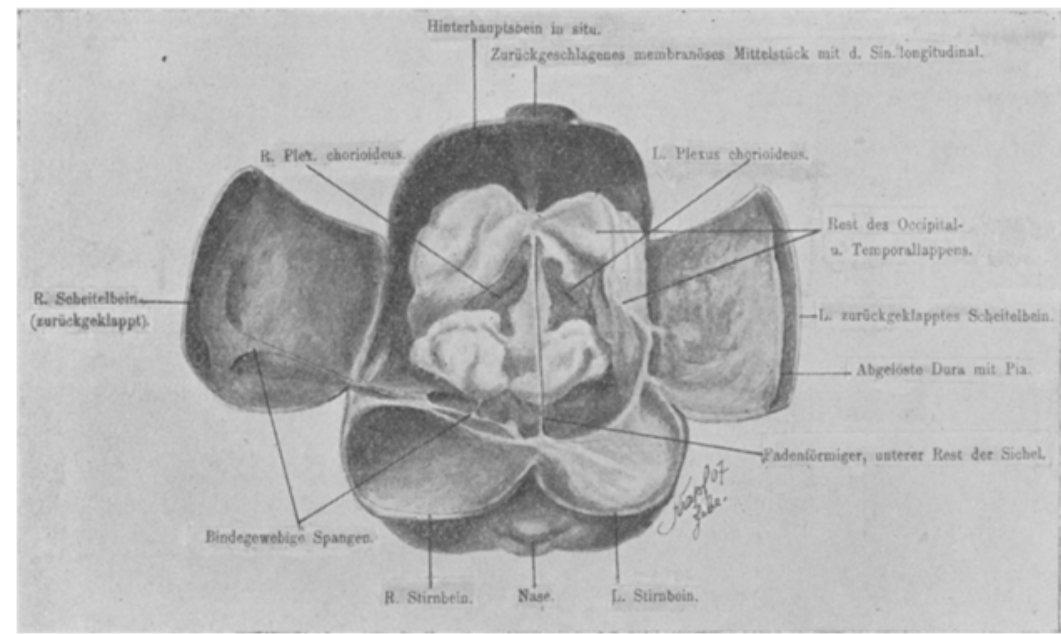

Ansicht von oben nach Zurückklappen der Scheitel- und Stirnbeine.

den Rest der Gehirnmasse nach hinten und mündet in der Gegend des Sinus confluens, er enthält den obliterirten Rest des Sinus longitud. inferior.

Auf dem Boden der vorderen Schädelgrube ist nichts mehr von Gehirn vorhanden; erst $31 / 2 \mathrm{~cm}$ nach rückwärts beginnt das Gehirn (Fig. 2); der Zwischenraum zeigt noch einige Spangen, die zum Theil obliterirte Gefässe enthalten. Der kleine noch vorhandene Theil des Gehirns beginnt an der Sella turcica, zeigt eine gleichmässig gelblich erscheinende glatte von einzelnen rundlichen Gruben durchzogene Oberfläche.

Die Gehirnmasse hat eine Länge ron $5 \mathrm{~cm}$ und weist an der 
höchsten Stelle über dem Tentorium eine Dicke von $1,5 \mathrm{~cm}$ auf. Beiderseits $1 / 2 \mathrm{~cm}$ von der Medianlinie entfernt liegen die beiden Plexus chorioidei und die beiden Seitenventrikel frei. Die Plexus haben eine Länge von $2 \frac{1}{2} \mathrm{~cm}$, sind gut entwickelt und weisen eine ockergelbe Farbe wie die Oberfläche des Gehirns selbst auf. Man sieht deutlich ihren Austritt aus dem 3. Ventrikel und kann ihre Einsenkung in das Vorderhorn gut feststellen. Die Decke des 3. Ventrikels ist erhalten als eine etwa $3 \mathrm{~mm}$ dicke Membran; nach ihrer Incision liegt der 3. Ventrikel mit dem Adergeflecht frei, doch zeigt dies die gewöhnliche Farbe, nicht das gelbe Colorit. Auf den ersten Blick bereits fällt auf, dass die Wände der grossen Höhle eine merkwürdig ockergelbe Farbe zeigen. Ueber dem Stirn- und dem Hinterhauptsbein tritt dieses Verhalten weniger scharf herror, sehr stark ist die Farbe üher den noch erhaltenen Gehirnpartien, an dem Plexus chorioideus und über dern linken Scheitelbein. Dem linken Scheitelbein haftet eine etwa $1 / 2 \mathrm{~mm}$ dicke gelbliche Schicht an. Bereits die makroskopische Betrachtung macht die Annahme wahrscheinlich, dass es sich um Färbung durch Blutpigment handelt, die mikroskopische bestätigt die Annahme.

Von grösster Wichtigkeit ist das Verhalten der Meningen. Die Dura lässt sich überall, wie beim Neugeborenen stets, etwas schwer vom Knochen abziehen, sie zeigt keine Verdickung, in den Furchen der Scheitelbeine sieht man die Art. meningeae regelmässig verlaufen. Auch die histologische Structur der harten Gehirnhaut zeigt keine Abweichung von der Norm. Es ist demnach eine Pachymeningitis mit Sicherheit auszuschliessen.

Die Pia liegt der Dura innig an, sie erscheint verdickt, in ihr befindet sich, namentlich über dem linken Scheitelbein das Blutpigment eingelagert. Von der Dura lässt sie sich ohne Schwierigkeiten abziehen.

Die mikroskopische Untersuchung der Pia (Fig. 3) ergiebt folgenden interessanten Befund: Nach aussen liegt die Pia, sie enthält sehr reichliches Blutpigment in ihren Maschen, sonst ist jedoch ihre Structur ganz regelmässig, insbesondere fehlt jedes Zeichen einer Entzündung, keine kleinzellige Infiltration, keine Bacterien. Nach innen zu lagert ihr eine manchmal breitere, manchmal schmälere Schicht auf, an manchen Stellen fehlt sie auch vollständig. Sie enthält die für die Grosshirnrinde typische Anordnung und Form der Zellen, ohne eine Spur von Entzündung 
zu zeigen. Auch hier findet sich hier und da Finlagerung von Blutpigment.

Es kann sich nach diesem Befunde um nichts anderes handeln, als um den Ueberrest der Grosshirnrinde, der bei der makroskopischen Betrachtung ganz der Erkennung entgangen war.

An den meisten Stellen findet sich übrigens auch mikroskopisch keine Spur von Grosshirnsubstanz mehr.

Figur 3.

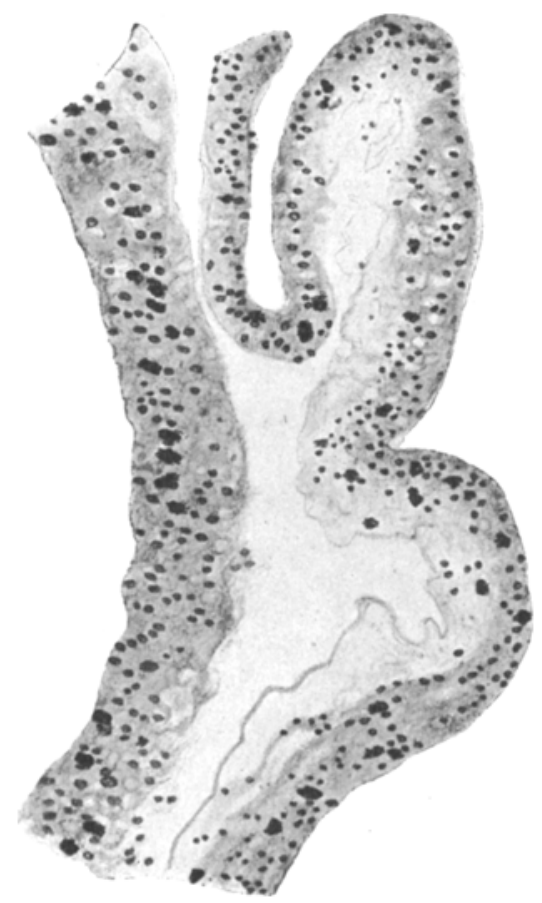

Die dunklen schwarzen Schollen sind Pigmentkörner.

Die mikroskopische Untersuchung der noch erhaltenen Theile des Grosshirns ergiebt, lass die der Höhle anliegenden Stellen stark von Blutpigment durehsetzt sind, während sich in den tiefer gelegenen Partien das schollige Pigment nur sehr selten findet. Die Zellen der Rind̉e und des Markes weichen in ihrem Bau und in ihrer Färbbarkeit nicht von dem gewöhnlichen Verhalten ab, Fettkörnchenzellen finden sich im Marke nicht vermehrt, Infiltration 
mit Leukocyten fehlt, auch um die Gefässe herum wird die kleinzellige Infiltration vermisst.

Die freiliegenden Plexus chorioidei weisen ein vorzüglich erhaltenes Epithel auf. Zwischen den Epithelien, z. Th. auch in den Epithelien selbst sieht man die gelben Pigmentschollen eingelagert, wie ein gelber Kranz nmgiebt das Pigment die Oberfläche, auch in den äusseren Theilen des Bindegewebes findet sich noch etwas Pigment, tiefer nach innen wird es fast völlig vermisst. Die Blutgefässe sind nicht so strotzend mit Blut gefüllt, wie man dies sonst vorfindet, sie sind meistens collabirt, kleinere z. Th. auch obliterirt. Anzeichen von Entzündungen und Bakterien fehlen.

Auch die übrigen Organe des Körpers erfahren eine mikroskopische Durchsuchung. Die Lungen zeigen zum grösseren Theil das typische Bild der Atelektase, von Pneumonia alba njchts vorhanden, die Nieren und die Milz ohne Besonderheiten. In der Leber finden sich noch Blutbildungsherde.

Es wurde bei der mikroskopischen Untersuchung aller Organe namentlich auch auf syphilitische Veränderungen geachtet, es konnte jedoch gar nichts aufgefunden werden; auch das Wegner'sche Zeichen war negativ. Die Placenta, deren ungewöhnlich grosses Gewicht $(770 \mathrm{~g})$ auf Lues hinweist, konnte leider nicht untersucht werden, da sie entfernt worden war; auch erfährt das erhöhte Gewicht durch die Infarkte (wiederholte Blutungen) eine hinreichende Erk]ärung.

Fassen wir die Befunde noch einmal kurz zusammen:

Ein nicht ganz ausgetragenes Kind wird nach leichter und spontaner Geburt asphytisch geboren, bleibt trotz Wiederbelebungsversuche blau und stirbt nach 5 Stunden, ohne dass sich auffallende Erscheinungen von Seiten der Centralorgane (Lähmungen, Krämpfe) gezeigt hätten.

Die Autopsie ergiebt eine ausgedehnte Atelektase der Lungen und eine hochgradige Zerstörung des Grosshirns in seinen vorderen und mittleren Partien. Die Zerstörung betrifft zunächst die Marksubstanz, hat aber sekundär auch die Rindensubstanz ergriffen und dieselbe auf eine dünne Schicht reducirt. Stammganglien, Pons, Kleinhirn und Medulla oblongata sind dabei intact.

Es erhebt sich zunächst die Frage: Wie kam diese hochgradige Zerstörung zu Stande?

Zunächst wird man an einen Hydrocephalus internus 
denken. Congenital kommt Hydrocephalus bekanntlich nicht gerade selten in verschieden hochgradiger Ausbildung aus. In allen Fällen ohne Austiahme tritt dabei durch die Erhöhung des intracraniellen Druckes eine Erweiterung der nachgiebigen Schädelkapsel auf, die sich durch weite Fontanellen, klaffende Nähte, grossen Kopfumfang offenbart. Wenn es sich um Hydrocephalus gehandelt hätte, der, wie neuere Untersuchungen lehren, häufig auf entzündlicher, namentlich luetischer Grundlage sich entwickelt, so wäre kein Grund einzusehen, warum gerade hier die so charakteristische Veränderung des Schädels ausgeblieben ist. Auch wäre nicht der Schwund der Sichel, die ejgenthümlich wellige Oberfläche des Grosshirnrestes, das Ueberbleiben der verschiedenen Spangen $\mathrm{zu}$ erklären. Dass erhöhter Innendruck thatsächlich gefehlt hat, geht auch aus dem Umstand hervor, dass die Hirnwindungen der noch erhaltenen hinteren Gehirnpartien ihre regelmässige Ausbildung haben.

Differentialdiagnostisch kommt weiter eine Meningitis und von der sich fortsetzend eine Encephalitis in Betracht.

Es fehlen jedoch alle Charakteristika sowohl einer Leptomeningitis wie einer Pachymeningitis. Die Dura weist makro- wie mikroskopisch normale Verbältnisse auf, die Pia ist zwar etwas verdickt, doch kommt die Verdickung nur durch die starke Infiltration mit Blutpigment. Jede Spur einer Entzündung fehlt; keine kleinzellige Infiltration, keine Bakterien; auch Reste einer alten Entzündung werden vermisst.

Wir werden daher zu der Annahme gedrängt, dass der Process, der zu der weit gehenden Zerstörung des Gehirns geführt hat, innerhalb der Gehirnsubstanz selbst stattgefunden haben muss. Da die Grosshirnrinde zum Theîl noch erhalten ist, wenn auch recht erheblich reducirt, so kann man den Process noch weiter lokalisiren und sagen, dass sein primärer Sitz in der Marksubstanz oder in den Gehirnventrikeln gewesen sein muss.

Hierfür kommen nur 2 Dinge in Betracht: Entzündung oder Haemorrhagie.

1. Entzündung. Zum erstenmale ist von Virchow (1865) über eine Gehirnerkrankung Neugeborener und junger Kinder berichtet worden, die er mit dem Namen Encephalitis interstitialis congenita belegte und von der er zwei Formen untersehied: eine diffuse und eine herdförmige Erkrankung. Der Process war hauptsächlich lokalisirt in der Marksubstanz beider Grosshirn- 
hemisphären, besonders im Gebiete der Balkenstrahilung, aber auch im Balken selbst, oft im Bereiche der Rückenmarksstränge, nur ganz ausnahmsweise im Kleinhirn und Medulla oblongata; stets frei war die Hirnrinde. Es handelt sich nach der Anschauung von Virchow um einen entzündlichen Process in dem interstitiellen Gewebe, in der Stützsubstanz des Gehirns, der charakterisirt ist durch Auftreten von Fettkörnehenzellen, die entweder diffus die Hirnmasse durchsetzen oder aber in gelbweissen schon makroskopisch erkennbaren Herden von Stecknadelkopf- bis Erbsengrösse auftreten. Wenn die Herde unter sich confluiren, so kann es zu einer weitgehenden Zerstörung des Hirnmarkes kommen. Diese Encephalitis interstitialis findet sich hauptsächlich bei Kindern, die an acuten Exanthemen (Variola) und septischen Processen versterben, auch Lues und vielleicht auch ischoröse Processe spielen eine Rolle.

Der Fall, den Virchow zum Ausgangspunkt seiner Untersuchungen genommen hat and den er in extenso mittheilt, ist folgender:

Eine ganz gesunde (nicht luetische) Erstgebärende kommt spontan mit einem ausgetragenen Kinde nieder. Die Mutter erkrankt an Wochenbettfieber, das bis zum 12. Tage anbielt. Das Kind nahm anfangs die Brust nicht, später jedoch trank es; es wurde sonst nichts Auffälliges an ihm bemerkt, nur war es ungewöhnlich ruhig. Am 6. Tage starb das Kind plötzlich.

Section: Jeterus, mehr cyanotische Verfärbung des Bauches und der Unterextremitäten. Pia leicht ödematös und etwas hämorrhagisch infiltrirt, die Gefässe stark gefüllt. Die graue Substanz der Rinde an diesen Stellen von bräunlicher Färbung. Auf Durchschnitten durch die Grosshirnhemisphären zeigt sich ein weicher, fast breiiger Zustand beinahe der gesammten weissen Substanz, welche ein schmutzig graurothes Aussehen darbietet; nur die Corpora striata, die Thalami und Corpora quadrigemina stehen als feste Theile hervor, sehen aber wie die grauen Cortikalschichten blass und weisslich aus. Pons und Kleinhirn scheinbar normal, namentlich auch die weisse Substanz an ihnen nicht verändert. Die mikroskopische Untersuchung ergab, dass die weisse Substanz des Gehirns durch und durch voller Fettkörnchenkugeln war, neben denen hie und da kleine Gruppen gewucherter Zellen lagen.

In gewissen Gegensatz zu Virchow setzten sich die nächsten Bearbeiter dieser Frage. Zunächst untersuchte Hayem Gehirne von 12 Kindern, die an verschiedenen Krankheiten verstorben waren, und fand stets Fettkörnchenzellen in theils diffuser, theils herdförmiger Anordnung. Die Schlussfolgerunden Virchow's sind ihm zu weitgehend.

Parrot brachte den Process in Zusammenhang mit der 
Athrepsie, die ihn einmal durch Inanition, dann aber auch durch die bei Athreptischen veränderte Blutbeschaffenheit herbeiführen könne. Die gleichen Veränderungen konnte er bei hungernden Katzen und Sperlingen experimentell erzeugen. Es handele sich nur um Nekrobiose, nicht um eine Entzündung.

Jastrowitz, der eine grössere Anzahl von Gehirnen Neugeborener und Kinder untersuchte, kommt zu dem Resultate, dass die Fettkörnchenzellen sowohl in diffuser als auch in circumscripter Form eine normale Erscheinung sind und dass sie mit der Entwicklung des Centralnervensystems in Beziehung stehen, eine Ansicht, der auch Flechsig zustimmte. Nur wenn die Fettkörnchenzellen ausserhalb der gewöhnlichen Fundorte und jenseits eines gewissen Alters vorkommen, haben sie nach der Meinung von Jastrowitz eine pathologische Bedeutung.

Auch Kramer, der vergleichende Untersuchungen an Föten, Neugeborenen und jungen Säuglingen anstellte, ist der Ansicht, dass nur ein massenhaftes Auftreten zu einer Störung der Gehirnfunktion führe und pathologisch sei.

v. Limbeck hält die diffuse Infiltration mit Fettkörnchen für eine normale Erscheinung, dagegen ist das herdförmige Auftreten der Zellen pathologisch und beruht im Sinne von Virchow auf einem irritativ-entzündlichen Process. Durch die Confluenz kleiner Herde erklärt er die Entstehung eines grossen hämorrhagischen Erweichungsherdes im Bereiche der Stirn-, Scheitel- und Schläfenlappen und in den Centralganglien.

Da dieser Fall eine gewisse Aehnlichkeit mit dem unsern hat, soll hier etwas genauer auf ihn eingegangen werden.

Es handelte sich um das Kind einer Zweitgebärenden, das bei der Geburt $48 \mathrm{~cm}$ lang war und $2800 \mathrm{~g}$ wog. Während der 4 tägigen Lebenszeit fiel nur auf, dass seine Bewegungen immer sehr träge waren und dass es selbst auf gröbere mechanische Hautreize fast gar nicht reagirte. Ausgesprochene Lähmungen bestanden nicht. Am 4. Tage starb es plötzlich. Die Autopsie ergab folgenden Befund: Der Schädel zeigt den gewöhnlichen Umfang und regelmässige Verknöcherung, die Meningen sind sehr blutreich und liegen der Hirnrinde innig auf, das Grosshirn hat eine weiche, schwappende Beschaffenheit, die Rinde zeigte regelmässige Furchenbildung. Fast der ganze rechte Scheitellappen, der obere Theil des Schläfenlappens und die hintere Partie des Stirnlappens waren durch einen Defect der Hirnsubstanz substituirt, über welche die innere Meningen brückenartig hinüberzogen, sodass die genannten Hirnpartieen durch ein cystenartiges Gebilde vertreten zu sein schienen, an dessen innerer Fläche man noch stellenweise sehr deutlich Reste von Hirnsubstanz wahrnahm. Die Cyste war mit einer bräunlich-rothen, missfarbigen, mit Flocken untermengten Flüssigkeit 
angefüllt. Links war der Defect noch grösser, auch war hier noch die Marksubstanz des Hinterhauptslappens zerstört. Das Kleinhirn, die Pons Varoli und die Med. oblongata zeigten makroskopisch keine besondere Veränderungen.

v. Limbeck berichtet im Anschluss an den ersten Fall noch über eine zweite Beobachtung: ein $21 / 2$ jähriger, imbeciller, hydrocephalischer Knabe zeigt eine Porencephalie, die in ihrer Ausdehnung eine Aehnlichkeit mit der Höhle des ersten Falles hat; er ist geneigt, diese Porencephalie auf dieselbe Ursache, nämlich auf eine congenitale Encephalitis wie bei Fall 1 zurückzuführen.

Es handelte sich also in der ersten Beobachtung von v. Limbeck um eine ziemlich ausgedehnte Zerstörung der Marksubstanz, am Besten war das Mark noch in dem Stirnlappen erhalten, an anderen Stellen war die Zerstörung bis zur Rinde gediehen.

Weder in den Gefässen der Convexität noch in den basalen Gefässen liess sich ein Thrombus oder eine Endarteriitis nachweisen. Auch die mikroskopische Untersuchung der Gefässe ergab ein völlig negatives Resultat.

In der die Höhle amgebenden Gehirnsubstanz fand sich eine "starre Infiltration mit partieller Nekrotisirung der Gewebe. „Die von den erweiterten Seitenventrikeln entfernter liegenden Hirnpartien waren von überaus zahlreichen Herden kleinzelliger Infiltration durchsetzt ", zwischen denen man Fettkörnchenzellen fand.

Von einer Untersuchung auf Bakterien wird nichts erwähnt. Nach den Befunden kann es nicht zweifelhaft sein, dass es sich hier um wirklich entzündliche Vorgänge und eine erst vor Kurzem eingetretene hämorrhagische Erweichung des Gehirns gehandelt hat. Ob dabei der Fall des Kindes auf den Boden - es handelte sich um eine Sturzgeburt, am Kinde liess sich weder äusserlich, noch am Knochen eine Verletzung feststellen - so gleichgiltig gewesen ist, wie Limbeck meint, erscheint fraglich.

Bei den Beobachtungen Fischl's handelte es sich nicht um congenitale Encephalitis, sondern um Encephalitis, welche erst mehr oder minder lange Zeit nach der Geburt auftrat. Interessant ist namentlich ein ausführlich mitgetheilter Fall: Ein nicht ganz ausgetragenes Kind, das am 12. Lebenstage unter Temperatursteigerungen und cerebralen Symptomen erkrankte und am 52. Lebenstage zu Grunde ging, zeigte bei der Sektion eine völlig breiige Erwei- 
chung des Marklagers, die Grosshirnhemisphären waren in zwei schlaffe Säcke umgewandelt, deren Wandungen von dem Reste der Rinde gebildet wurden. Kleinhirn und Med. obl. von gewöhnlicher Consistenz. Bakterien fanden sich in Schnitt und Cultur nicht, dagegen starke Leukocyteninfiltration, namentlich um die Gefässe herum.

In einer anderen Beobachtung Fischl's ging das Kind an einer septischen Infektion zu Grunde, im Bereiche der Marksubstanz des Grosshirns zeigten sich kleine gelbgraue Herde, die sich als Leukocytenhaufen, im Bereiche welcher mikroskopisch culturell Staphylococcus pyogenes albus sich vorfand, erwiesen und als Metastasen des septischen Processes bezeichnet werden mussten.

In einer späteren Mittheilung, in der über 6 weitere Fälle berichtet wird, betont Fischl, dass es sich um wirkliche Entzündungsherde handele; dies werde viel sicherer als durch die Fettkörnchenzellen durch den Nachweis leakocytärer Elemente und der Bakterien festgestellt. Thatsächlich seien die circumscripten Herde Virchow's gar nichts anderes als eine Leukocyteninfiltration, die Leukocyten haben durch die fettige Degeneration nur die Form der Fettkörnchenzellen angenommen. Die Gliafasern seien wahrscheinlich auch daran betheiligt, in welcher Weise, sei noch nicht genau $\mathrm{zu}$ bestimmen. Fischl weist ferner darauf hin, dass die encephalitischen Herde in Beziehung zu den Gefässen stehen, „indem sich theils halbmondförmig an, theils circulär um ein Gefäss gelagerte Leukocytenhaufen vielfach in der Marksubstanz vorfinden." Manchmal bestehe auch eine stärkere Gefässfüllung. Nur in einem einzigen Falle konnten Bakterien in der Gehirnsubstanz im Schnitt nachgewiesen werden, in einigen anderen Fällen wurden sie, trotzdem die übrigen Organe Zeichen septischer Erkrankung mit positivem Bakterienbefunde aufwiesen, vermisst. Culturen aus der Gehirnsubstanz wurden nicht angelegt.

Fischl kommt zu dem Schlusse, dass die Herde bei der Encephalitis entzündlicher Natur sind, die einmal „metastatisch als Theilerscheinung septischer Allgemeininfektion, ein anderes Mal auf toxischem Wege als Effekt von durch die Sepsiserreger producirten Giftstoffen zu Stande kommen, während in einem Theile der Eälle keine derartige Ursache sich nachweisen Jässt."

Für den bereits oben genauer citirten Fall, in dem sich weder durch Cultur noch im Schnitt Bakterien nachweisen liessen, nimmt Fischl an, dass die Zerstörung des Markes durch Toxin- 
wirkung zu Stande gekommen sei und zwar stamme das Gift aus dem Darme. Der Fall weicht insofern auch von der Encephalitis congenita $a b$, als nicht in der Marksubstanz, sondern in der Rinde die dichteste Infiltration mit Leukocyten und Verdrängung der specifischen Elemente bestand, im Mark dagegen Blatung und Nekrose. Die Meningen, von denen gelegentlich ebenfalls encephalitische Processe ihren Ausgang nehmen, erwiesen sich als vollständig freị.

Die neuesten Untersuchungen über die Encephalitis congenita im Sinne Virchow's stammen von Thiemich, der mit der sehr leistungsfähigen Marchi'schen Methode arbeitete.

Er findet: "Die diffuse Encephalitis betrifft nie ganz gleichmässig alle markhaltigen Theile des Gehirns, sondern meist die central gelegenen stärker als die Markleisten der einzelnen Windungen, am stärksten immer den Balken und den Fornix." "Im Hemisphärenmark ist das Auftreten von Körnchenzellen meist vergesellschaftet mit deutlich erkennbarem Markscheidenzerfall, den man aber gleich intensiv an anderen Stellen desselben Gehirns auch ohne die Anwesenheit der Körnchenzellen finden kann. ${ }^{6}$ Es hängt daher das Auftreten der Körnchenzellen nicht nothwendig mit dem Markscheidenzerfall zusammen. Er ist der Ansicht, dass man ndie Encephalitis interstitialis wenigstens in ihren leichteren Graden nach der Häufigkeit, mit der man sie an den Leichen auch kräftiger ausgetragener Kinder, die nach normal verlaufenem Partus durch einen Unglücksfall oder dergl. umgekommen sind, zu finden pflegt, keine für das betreffende Individunm so schwer wiegende Bedeutung besitzt, als Virchow anfangs anzunehmen geneigt war."

Ich musste auf die Frage der congenitalen Encephalitis hier näher eingehen, weil von Virchow und von anderen Autoren, so von Limbeck für seinen Fall angenommen wird, dass durch Confluenz von einzelnen kleinen encephalitischen Herden weitgehende Zerstörungen der Marksubstanz wie in unserer Beobachtung eintreten kann.

Fassen wir die Ergebnisse der neueren Untersuchungen äber die Encephalitis interstitialis congenita nach einmal kurz zusammen, so steht fest, dass es sich bei ihr nicht um etwas Einheitliches handelt, sondern um ganz verschiedenartige Processe. Es ist daher zweckmässig, die verschiedenen Formen scharf voneinander zu trennen: 
1. Die Form, in denen Fettkörnchenzellen in diffuser oder herdförmiger Anordnung im Mark des Grosshirns auftreten; sie findet sich bei sehr vielen kranken und gesunden Föten, Neugeborenen und jungen Säuglingen, jedoch nicht immer (Thiemich). Die einen Beobachter, so namentlich Jastrowitz, fassen das Auftreten der Fettkörnchenzellen als eine psysiologische Erscheinung auf, andere halten sie zwar für pathologisch, wie der neueste Beobachter, Thiemich, sprechen ihnen jedoch jede klinische Bedeutung ab. $\mathrm{Zu}$ grösseren Erweichungsherden kommt es nie, die Genese des Vorgangs ist nicht näher bekannt.

2. Die zweite Form ist sicher echt entzündlicher Natur und führt zu weitgehenden Zerstörungen der Gehirnsubstanz.

Nur diese zweite Form kommt für die Erklärung unserer Beobachtung in Betracht.

Virchow und v. Limbeck nehmen für ihre Beobachtungen mit weitgehender Zerstörung der Marksubstánz einen bereits intrauterin einsetzenden, also einen congenitalen Process an. Ein Beweis für diese Annahme ist jedocb bei keinem der Fälle erbracht.

Zunächst der Fall von Virchow.

Ein vorher scheinbar gesundes Kind stirbt am 6. Tage unvermuthet, die Autopsie ergiebt eine haemorrhagische Erweichung des Marks offenbar sehr frischen Charakters und verschiedene andere Entzündungserscheinungen an den Hirnhäuten und an der Hirnsubstanz selbst. (cfr. Beschreibung S. 710.) Die Mutter liegt mehrere Tage an Wochenbettfieber - damals hiess es noch Milchfieber - krank. Bei dieser Sachlage ist es mehr wie wahrscheinlich, dass auch das Kind an einem septischen Process erkrankt und erlegen ist. Gerade diese machen bei Neugeborenen häufig nur geringe Erscheinungen und gerade bei ihnen kommt es, wie die Untersuchungen Fischl's dargethan haben, mit Vorliebe zu Erweichungen und Einschmelzungen der Marksubstanz.

Bei der Beobachtung Limbeck's handelt es sich ebenfalls um entzündliche Vorgänge; dafür spricht die Hyperaemie der Gehirnsubstanz, die starke Leukocyteninfiltration und die Nekrose des umgebenden Gewebes. Der Erweichungsprocess ist noch sehr jungen Datums; denn die erweichte Masse zeigt eine trübe haemorrbagische Beschaffenheit, die Höhle fetzige und unregelmässige Wandungen. Von dem Befinden der Mutter ist leider nichts angegeben. Das Kind ist durch Sturzgeburt zur Welt ge- 
kommen und stürzte mit dem Kopfe voran auf den Boden. Wenn auch an der Haut und an den Knochen eine Verletzung nicht nachzuweisen war, so liegt doch die Annahme nicht ferne, dass durch das Trauma eine Verletzung intracranieller Blutgefässe stattfand und das gebildete Haematom nachträglich der Infection verfiel. Da das kind volle vier Tage am Leben blieb, könnte eine acquirirte Encephalitis auf septischer Grundlage die Zerstörung des Markes herbeigeführt haben. Jedenfalls liegt auch für den Fall von Limbeck die Annahme einer acquirirten Anomalie viel näher.

Es sind hauptsächlich die schönen Beobachtungen von Fischl, welche auch bei den vorgenannten Fällen einen postnatalen Process wahrscheinlicher machen. Bei Fischl handelt es sich um Kinder, die erst Tage und Wochen nach der Geburt an den encephalitischen Symptomen erkrankten und bei denen in der Mehrzahl der Fälle Streptokokken oder Staphylokokken nachgewiesen werden konnten. Die anatomisch-pathologischen Befunde am Gehirn haben mit den angeblich congenitalen Fällen Virchow's und Limbeck's zum Theil sehr grosse Aehnlichkeit. 1a auch bei den zwei genannten Beobachtungen entzündliche Erscheinungen bestanden (starke Leukocyteninfiltration), erscheint die Annahme berechtigt, dass auch hier septische Keime im Spiele waren.

Wir kommen zu der Erkenntniss, dass die angeblich congenitalen Fälle von Encephalitis mit weitgehender Zerstörung der Gehirnsubstanz im Sinne von Virchow garnicht congenital sind, sondern erworbene Infectionen meist septischer Natur darstellen.

Unser Fall ist jedoch fraglos congenital. Er hat demnach eigentlich nichts mit den vorgenannten Processen zu thun.

Allein es lässt sich auch direct der Beweis führen, dass beide Processe genetisch grundverschieden von einander sind.

Während in den Fällen sogenannter "congenitaler" Encephalitis stets entzündliche Erscheinungen bestanden, zum Theil sogar septische Keime nachgewiesen wurden, fehlt dagegen bei unserem Falle jede Spur einer Entzündung: keine kleinzellige Infiltration, keine Hyperaemie, keine Bakterien.

Es wäre ein vollständiges Novum und stände im schroffsten Gegensatz zu allen bisherigen Erfahrungen in der Geburtshilfe, wollte man annehmen, dass bei einer während der ganzen Schwangerschaft völlig gesunden Mutter septische oder anders geartete Keime durch die Placenta auf den Fötus übergehen. 
Anzunehmen, es handele sich um eine Intoxication, hat ebenso wenig Wahrscheinlichkeit. Woher sollen bei der sonst gesunden Mutter die Toxine kommen? Und warum sieht man denn solche Folgen der Intoxicationen so extrem selten?

Es liegt demnach den destructiven Processen im Hirnmark bei unserem Falle keine Entzündung und keine Infection zu Grunde. Es bleibt demnach nur die Gehirnhämorrhagie übrig.

\section{Die Gehirnhämorrhagie.}

Für diese Annahme haben wir verschiedene positive Anhaltspunkte.

Schon beim ersten Blick fällt die gelbe Farbe der ganzen Höhle auf. Auf der Pia des linken Scheitelbeins liegt eine etwa 1/2 mm dicke Schicht einer braungelben Substanz. Die mikroskopische Untersuchung und die mikrochemische Reaction mit Ferrocyankalium zeigt, dass es sich um Blatpigment handelt. Es ist die Pia, es ist die Oberfläche der Hirnpartieen; die die Wand der Höhle bilden, es sind namentlich auch die Plexus chorioidei wie imprägnirt mit dem Blutpigment.

Eine derartige Menge von Blutpigment kann nụr als ein Residuum einer grösseren Blutung gedentet werden. Aus einer einfachen haemorrhagischen Erweichung würden wohl nie derartige Anhäufungen von Blutpigment restiren.

Nach der glatten Beschaffenheit der Wandungen der Höhle, nach der geringen Trübung der Höhlenflüssigkeit und dem Aussehen des Pigmentes zu schliessen, muss die Blutung schon längere Zeit zurückliegen.

Man wird nicht fehl gehen, wenn man das im 4. Monate der Schwangerschaft erlittene Trauma mit der Entstehung der Blutung in ursächlichen Zusammenhang bringt. Um diese Zeit ragt der Fundus uteri bereits über die Symphyse herauf, er wurde beim Sturze auf die Schüssel (3 Stufen tief) direct von der Gewalt getroffen; der reichliche Blutabgang unmittelbar nach dem Falle weist ebenfalls auf eine starke mechanische Schädigung hin. Bei dem ersten Sturze, der bereits nach dem erstmaligen Ausbleiben der Periode erfolgte, ist die Gehirnhämorrhagie wohl nicht erfolgt, es hätten sich sonst noch weitergehende Veränderungen und wohl auch Missbildungen am Schädeldach gezeigt. 
Nach der starken Anhäufung des Blutpigmentes unter dem rechten Scheitelbeine hat es den Anschein, als ob hier der Hauptsitz der Blutung gewesen wäre; durch Erhöhung des intracraniellen Druckes entstand Anämie und collaterales Oedem nnd die Substanz verfiel bis auf spärliche Reste von Rinde der Erweichung. Schliesslich resultirte daraus die grosse Cyste, wie sie bei der Section gefunden wurde.

Dass gerade die vorderen Theile des Grosshirns zerstört wurden, dagegen die hinteren zum Theil und Kleinhirn und Medulla oblongata ganz erhalten blieben, erklärt sich aus dem Sitze der Blutung; die näher gelegenen Hirnpartien leiden unter dem Drucke stets mehr als entferntere. Kleinhirn und verlängertes Mark zumal sind durch das Tentorium vor grösserem Drucke geschützt.

Besonders leicht verfällt die Marksubstanz beim Fötus und Neugeborenen der Erweichung, wie die angeführten Fälle aus der Literatur beweisen. Es ist daher auch bei der Blutung in unserem Falle nicht zu verwundern, dass am ausgedehntesten das Mark zerstört wurde, die Rinde sich stellenweise wenigstens noch in spärlichen Resten erhielt.

Soweit ich die Literatur einsehen konnte, ist dies der erste Eall, bei dem durch ein Trauma der Mutter (Sturz auf den Leib im 4. Monat) eine schwere cerebrale Blutung erfolgte, die zu einem fast das ganze Grosshirn umfassenden Gehirndefect führte.

Auch in klinisch-geburtshülflicher Beziehung hat die Beobachtung ein gewisses Interesse. Trotz des riesigen Hirndefectes entwickelt sich das Kind intrauterin gut, zeigt bei der Geburt regelmässige Herztöne. Diese Erscheinung ist nicht auffällig, da die Medulla oblongata ganz intact erscheint. Erst als nach der Geburt die Athemthätigkeit einsetzen soll, versagt die Medulla oblongata, vielleicht in Folge veränderter intracranieller Druckverhältnisse ihren regelmässigen Dienst, die Athmung ist nicht in Gang zu bringen und das Kind erliegt nach 5 Stunden. Durch die Geburtsvorgänge war die merkwürdige Asphyxie in keiner Weise zu erklären.

Auch für die Genese späterer Porencephalien ist die Beobachtung von Bedeutung. Man denke sich die Zerstörung des Gehirns innerhalb engerer Grenzen, ein Theil des Markes und die Rinde erhalten, so hätte dieses Kind am Leben bleiben können 
und hätte späterhin entsprechende Defecte gezeigt. Und das bei einem Kinde, das sonst gesund ist und von gesunden Eltern stammt!

Leider sind genauere Beobachtungen über das Verhalten der Motilität und Sensibilität bei dem Kinde während der kurzen Zeit des Lebens nicht angestellt worden, auffallende Erscheinungen hat es nicht gezeigt.

\section{Zusammenfassung.}

1. Eine wirklich congenitale, durch intraterine Entzündungsprocesse im Sinne von Virchow's Encephalitis circumscipta erfolgte Zerstörung des Grosshirnmarkes

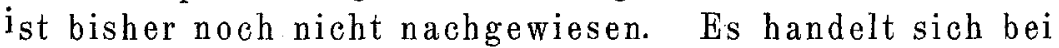
den bisher als Encephalitis congenita beschriebenen Hirndefecten um Zustände, die sub partu durch cerebrale Hämorrhagien oder extrauterin durch septische Infectionen, vielleicht auch Intoxicationen entstanden sind.

2. Bereits intrauterin kann es durch eine Blutung in die Ventrikel oder in die Hirnsubstanz zu weitgehenden Einschmelzungen des Grosshirnmarks, secundär auch der Rinde kommen, so dass daraus grosse Hirndefecte (Porencephalien), in unserem Falle Zerstörung fast des ganzen Grosshirns resultiren. Der Schädel kann dabei regelmässige Form und Knochenentwicklung aufweisen.

\section{L i t e r a tur.}

Fischl, R., Ein Beitrag zar Kenntniss der Encephalitis beim Säugling. Prag. med. Wochensehr. 1897. No. $26 . \quad$ S. 307.

Derselbe, Zur Kenntniss der Encephalitis beim Sängling. Jahrbuch f. Kinderheilkunde. Bd. 49 . H. 1. S. 58. 1899.

Gangh ofner, Ueber cerebrale spastische Lähmungen im Kindesalter. Jahrbuch f. Kinderheilk. Bd. 40. H. 2 u. 3. S. 219.1895.

Hayem, Etudes sur les diverses formes d'encéphalite. Paris. De la Haye. 1868. S. 77 .

Zastrowitz, Arch. f. Psychiatrie und Neurologie. 2, 3. und Berlin. klin. Woohensehr. 1883. S. 70. No. 46 .

Kramer, Ueber das Vorkommen von Körnchenzellen im Gehirn Neugeborener. Dissert. Berlin. 1885.

Limbeck, Zur Kenntniss der Encephalitis congenita u. ihrer Beziehung zur Porencephalie. Zeitschr. f. Heilkunde. Bd. VII. Heft 2 u. 3. S. 87. 1886. 
720 Seitz, Ueber die durch intrauterine Gehirnhämorrhagien u.s.w.

Parrot, Arch. de physiol. normale et pathol. I. 1868. p. 530 und Atrepsie, Leģons recueillies par le Dr. Troisier. Paris. Masson. 1877. p. 291. Reymond, Ueber Encephalitis diffusa resp. Poliencephalitis des Kindes. Jahrbuch f. Kinderheilk. Bd. 44. Heft 2. S. 157. 1897.

Steffen, Encepholitis mit Erweichung in Gerhard's Handbuch d. Kinderkrankheiten. Bd. 5. Abth. I. Hälfte 2. S. 503.

Thiemich, Ueber die Schädigung des Centralnervensystems durch Ernährungsstörungen im Säuglingsalter. Jabrbuch f. Kinderheilk. Bd. 52. H. 5. S. 810.1900.

Virchow, Congenitale Encephalitis u. Myelitis. Virchow's Arch. Bd. 38. H. 1. S. 129.1867. 